Universidade Federal de Gaiás

REVISTA DO PROGRAMA
DE PÓS GRADUAÇÃO PROJETO CIDADE

Revista Jatobá, Goiânia, 2020, V.2 


\author{
Universidade Federal de Giás \\ REITDR: Eward Madureira Brasil \\ VICE-REITORA: Sandramara Matias Chaves \\ DIRETOR DA FACLLDADE DE ARTES VISUAIS: Braúlio Vinicius Ferreira \\ COLRDENADORA DO PRDGRAMA DE PD́S-GRADUAÇÃ̃ EM PRDJETD E CIDADE: Erika Cristine Kneib \\ REVISTA JATOBÁ \\ Revista de Pesquisas e debate em Arquitetura e Urbanismo
}

MISSÃD

Publicar estudas sobre os temas do planejamenta, projeta, teoria, história e crítica em Arquitetura e Urbanismo buscando a multidisciplinaridade e a interdisciplinaridade entre os campos do conhecimento da geagrafia, história, ciências sociais e ambientais, às engenharias, às artes e à filosafia com ênfase na originalidade no âmbito nacional e internacional.

\title{
EDUIPE EDITORIAL
}

\section{Editores - chefes}

Luana Miranda Esper Kallas - UFG| FAV JPPGPC

Fernando Mello - UFG| FAV JPPGPC

\section{Editor de Layout}

Bráulio Vinicius Ferreira - UFG| FAV

\section{Editores de Seção}

Christine Ramos Mahler - UFG| FAV JPPGPC

Karla Emmanuelle R. Hora UFG IEECA |CIAMB e PPGP

Pedro Henrique Gonçalves UFG - Regional Goiás

\section{Editores técnicos}

Catia Ana Baldaina da Silva

Felipe Velosa de Paula

\section{Conselho Editorial}

Alcilia Afansa de Albuquerque e Melo - UFCG|CAU-UAEC-CTRN- PGH Ana Carolina de Souza Bierrenbach - PPGAU|UFBA

José Clewton da Nascimenta - UFRN|DARQ

José Waldemar Tabacaw - UNISUL|DCHCELA

Radriga de Faria - UnB| PPGFAU

Wilza Gomes Reis Lopes -UFP川| DDCA e PRDDEMA

\section{DADDS INTERNACIONAIS DE CATALOGAÇ̃̃̃ NA PUBLICAÇ̃̃̃ (СIP)}

$\mathrm{GPT} / \mathrm{BC} / \mathrm{UFG}$

J39 Revista Jatobá: Revista do Programa de Pós Graduação Projeto Cidade [recurso eletrônico]/ Universidade Federal de Goiás, Faculdade de Artes Visuais (FAV). Vol. 1, n. 1 (2019) - Dados eletrônicos. Goiânia: Faculdade de Artes Visuais, 2020-

Sistema requerido: Adobe Acrobat Reader.

Modo de acesso: World Wide Web:

< https://www.revistas.ufg.br/revjat/issue/view/2190>

Anual.

Descrição baseada em: v. 2, n. 2 (2020)

ISSN versão digital: 2675-1119

1. Arquitetura. 2. Arte. 3. Geografia. 4. História. I. Universidade Federal de Goiás, Faculdade de Artes Visuais. 